\title{
Management international
}

International Management

Gestiòn Internacional

\section{--> Voir l'erratum concernant cet article}

\section{Herman Simon, Stéphan Guinchard, « Les Champions Cachés du XXI ${ }^{\mathrm{e}}$ Siècle ", Economica, 2012}

\section{Pascal Gaudron et Aziz Mouline}

Volume 18, numéro 2, hiver 2014

URI : https://id.erudit.org/iderudit/1024204ar

DOI : https://doi.org/10.7202/1024204ar

Aller au sommaire du numéro

Éditeur(s)

HEC Montréal

Université Paris Dauphine

ISSN

1206-1697 (imprimé)

1918-9222 (numérique)

Découvrir la revue

Citer ce compte rendu

Gaudron, P. \& Mouline, A. (2014). Compte rendu de [Herman Simon, Stéphan

Guinchard, « Les Champions Cachés du XXI ${ }^{\mathrm{e}}$ Siècle », Economica, 2012].

Management international / International Management / Gestiòn Internacional,

18(2), 209-213. https://doi.org/10.7202/1024204ar

Tous droits réservés (C) Management international / International Management / Gestión Internacional, 2014
Ce document est protégé par la loi sur le droit d'auteur. L'utilisation des services d’Érudit (y compris la reproduction) est assujettie à sa politique d'utilisation que vous pouvez consulter en ligne.

https://apropos.erudit.org/fr/usagers/politique-dutilisation/ 


\section{Herman Simon, Stéphan Guinchard, «Les Champions Cachés du XXI" Siècle», Economica, 2012}

\author{
PASCAL GAUDRON \\ Chaire de management \\ international Walter J.-Somers
}

\author{
AZIZ MOULINE \\ CREM UMR CNRS 6211 \\ Université de Rennes 1
}

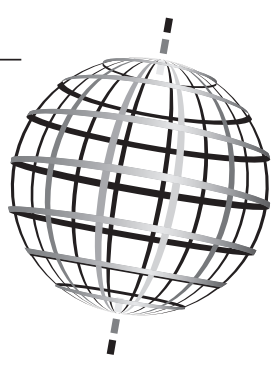

$\mathrm{D}$ epuis de très nombreuses décennies, les grandes entreprises font l'objet de plusieurs publications dans la littérature du management stratégique et de la théorie des organisations. Les facteurs, souvent invoqués pour expliquer cette situation - au-delà de la reconnaissance des noms des firmes et de leurs marques - résident dans la disponibilité des informations, l'accès aisé par les chercheurs aux variables économiques et financières, etc. Les spécialistes du management stratégique ont alors très souvent retenu ces grandes entreprises pour formuler des cadres théoriques que les générations suivantes complètent. Des manuels ont ainsi été consacrés à General Motors, IBM, Google, Facebook, LVMH, l'Oréal, Danone, etc.

Mais qui connaît ou a entendu parler d'Abrisud $\left(\mathrm{n}^{\circ} 1\right.$ européen des abris de piscine), de Babolat ( $\mathrm{n}^{\circ} 1$ mondial des cordages pour raquettes de tennis), de Look Cycle $\left(\mathrm{n}^{\circ} 1\right.$ mondial des cadres en carbone et pédales automatiques pour vélos), de TLD ( ${ }^{\circ} 3$ mondial des équipements d'assistante aéroportuaire), de Fischer ( $\mathrm{n}^{\circ} 1$ mondial des concentrés de fruit) ou bien encore de Pearl Clock ( $\mathrm{n}^{\circ} 1$ mondial des montres à quartz)? Il s'agit, on l'aura compris, de firmes appartenant à la catégorie des «Champions Cachés » émanant de pays aussi différents que l'Allemagne, la France, la Suisse, la Belgique, les Etats-Unis, le Canada, l'Afrique du Sud, l'Argentine, le Brésil, l'Inde, la Chine ou l'Egypte. Il est temps de s'interroger sur ces entreprises. H. Simon et S. Guinchard, (traducteur et associé français d'H. Simon) formulent d'ailleurs une remarque intéressante : est-ce que ces entreprises ne forment-elles pas des modèles bien meilleurs et bien plus instructifs que ceux inspirés par les grandes entreprises?

Il existerait donc une zone entre la très petite entreprise (TPE) et la grande entreprise (GE). Les PME sont souvent citées pour illustrer cette zone. En France selon l'INSEE, cette catégorie est «constituée d'entreprises qui occupent moins de 250 personnes, et qui ont un chiffre d'affaires annuel inférieur à 50 millions d'euros ou un total de bilan n'excédant pas 43 millions d'euros». Une autre catégorie doit cependant attirer notre attention : l'ETI (Entreprise de Taille Intermédiaire) telle qu'elle a été actée en mars 2008 en France dans la Loi de Modernisation de l'Economie. Elles ont entre 205 et 500 salariés, on en dénombre 4500 en France (10 000 en Allemagne) avec plus d'un quart de l'emploi salarié et un tiers des exportations. Elles réalisent un chiffre d'affaires compris entre 50 millions d'euros et 1,5 milliard et le total de bilan est inférieur à 2 milliards d'euros. Le rapport Gallois, remis au Président de la République à l'automne 2012, propose même une «stratégie ETI» $»^{1}$. Le ministre du commerce extérieur souhaite en identifier 800 afin de relancer les exportations. Leur rôle économique (développement des territoires, redynamisation de l'offre industrielle et conquêtes de marchés extérieurs) est donc reconnu.

Dans le groupe des ETI, il existe des entreprises qui méritent une attention particulière et une analyse approfondie. C'est justement le travail d'H. Simon et de S. Guichard dans leur ouvrage intitulé «Les Champions Cachés du $\mathrm{XXI}^{\mathrm{e}}$ siècle, Stratégies à succès », paru chez Economica en octobre 2012. Déjà en 1998, Hermann Simon avait axé son travail sur cette catégorie d'entreprises en montrant comment devenir le leader mondial quand on est une $\mathrm{PME}^{2}$. Il s'agit de la catégorie bien particulière intitulée «les Champions Cachés». Ce concept traduit les caractéristiques d'ETI peu connues du grand public alors qu'elles occupent pourtant l'une des trois premières places de leur marché national ou mondial. Elles «opèrent en amont des chaînes de valeur, fournissant des équipements, composants ou processus que l'on ne peut plus distinguer dans le produit ou le service final». En revanche, ces ETI sont bien connues de leurs clients, «leurs marques sont bien connues des acteurs concernés, leur réputation est exceptionnelle et leurs concurrents les prennent comme référence».

Les Champions Cachés existent partout dans le monde, mais pour les auteurs, en raison de certaines conditions (comme le lien entre le nombre de champions et les exportations), ils sont géographiquement localisés. Sur les 2000 recensées, les deux tiers se trouvent dans des pays germanophones. Les auteurs ont calculé que 1300 champions allemands, autrichiens et suisses réalisent plus d'un quart des exportations de ces pays. La France aurait entre 200 et 300 Champions Cachés. 
Pour être qualifié de Champion Caché, une entreprise doit remplir trois critères :

- Etre numéro un, deux ou trois de son marché à l'échelle mondiale (ou numéro un sur son continent),

- Réaliser un chiffre d'affaires inférieur à 3 milliards d'euros,

- Etre relativement peu connu du grand public.

Deux fortes caractéristiques sont également à noter : un taux de croissance annuel moyen de $8,8 \%$ et une part de marché - en moyenne de $33 \%$ - qui soit 2,3 fois celle de leur concurrent direct.

Au-delà de ces constations, il faut s'interroger sur les causes de l'excellence de ces Champions Cachés. La méthode et les résultats ne sont pas sans rappeler d'autres études, notamment celles de J. Collins et J. Porras ${ }^{3}$. Les auteurs indiquent avoir utilisé une liste constituée de 2000 entreprises du monde entier sur une durée de vingt cinq ans, en s'appuyant sur des informations publiques, les communications des entreprises, des enquêtes, des missions de conseil, des entretiens et des visites... et très peu la littérature du management en raison de l'absence d'études sur les Champions Cachés. Les auteurs démontrent «que beaucoup d'entreprises, parmi les plus compétitives, les plus réactives et celles qui rencontrent un succès durable, opèrent derrière un voile de discrétion et demeurent méconnues, même des experts du monde des affaires».

Les Champions Cachés sont avant tout des leaderships aux objectifs très ambitieux particulièrement quant à leur croissance et leur position sur leur marché. Une perspective de leadership général dans le secteur est fixée avec des dirigeants visionnaires. Certes, il y a évolution de la vision, affinement, confirmation a postériori et même des échecs, mais «le fait de formuler et, encore plus, le fait de vivre au quotidien de telles ambitions sont de puissants moteurs pour la réalisation».

Les auteurs ne trouvent pas de corrélation entre la taille et le taux de croissance des entreprises étudiées. Si les moyennes peuvent cacher des disparités, force est de constater que sur dix ans, le chiffre d'affaires des Champions Cachés a plus que doublé, avec des taux de croissance très similaires entre des entreprises pourtant de tailles très différentes. Les moteurs de la croissance sont multiples. Pour H. Simon et S. Guinchard, «l'internationalisation et l'innovation sont d'extraordinaires axes de développement, probablement dans cet ordre d'importance». L'innovation (à la fois sur des éléments techniques que des éléments de processus comme les processus de vente) est pour certains la principale source de croissance. Ils citent les cas de Brita avec les filtres à eau à usage domestique, Omicron avec ses

3. J. Collins, J. Porras, Built to Last - Successful Habits of Visionary Companies, New York, Harper Collins, 1994; J. Collins, Good to Great microscopes à effet tunnel ou encore Kärcher pour les équipements de nettoyage à haute pression.

Il faut signaler la dynamique qui touche les Champions Cachés qui ont des parts de marché importantes, mais «le désir de renforcement des positions de marché et le désir de poursuivre le développement de leur part de marché relative restent des puissants moteurs de croissance». Ainsi, ils augmentent parts de marché et consolident leur position de leader. Etre leader est même une caractéristique identitaire. Il faut noter que la position de leader ne se résume pas à un indicateur de volume ou de valeur pour ces Champions Cachés; ils sont leaders en termes de technologies et de qualité. Les autres facteurs sont la notoriété, la réputation et la tradition selon une trajectoire qui s'inscrit dans la durée confirmant ainsi «que la position du numéro un se construit sur une domination de long terme». D'ailleurs, les Champions Cachés le sont depuis près de 22 ans en moyenne. Les auteurs établissent ainsi une autre forte corrélation entre l'horizon à long terme des objectifs et la stabilité de l'équipe dirigeante (le PDG reste en moyenne 20 ans à son poste).

Les auteurs identifient par ailleurs le rôle déterminant de la focalisation comme caractéristique essentielle des Champions Cachés. L'objectif est de se concentrer sur un segment spécifique, surtout quand les ressources sont limitées. Ils définissent alors leur marché de façon étroite et y développent des positions très fortes voire dominantes. Certes, la forte focalisation est source de risques (en moyenne $70 \%$ du chiffre d'affaires est réalisé sur un marché) via la dépendance, la possibilité d'être attaqué par un autre produit et la taille réduite du segment. Les Champions Cachés semblent assumer cette position car «le choix de la simplicité, dans l'offre produit comme dans les groupes de clients ciblés, est l'une des clés du succès » et la gestion des risques de la focalisation «fait d'eux de grands innovateurs tout comme des concurrents féroces ». Trumpf, leader mondial des équipements de découpe laser, a bien connu cette ardente nécessité d'innover quand le laser a remplacé le découpage manuel. Il a été vigilant et a développé ses propres outils laser. Ainsi l'entreprise a protégé sa position de leader et est devenu une firme très performante sur ce segment.

La diversification vers d'autres marchés et d'autres produits semble bien se développer parmi les champions cachés de grande taille. Au-delà de la diversification des risques, il s'agit «d'anticiper les freins à la croissance attendus dans le proche avenir». Forte part de marché et marché étroit se conjuguent difficilement avec la croissance du chiffre d'affaires. L'exploitation des compétences est aussi un moteur reconnu pour amorcer la diversification comme le montre l'exemple de l'entreprise Trumpf qui a appliqué son expertise au secteur médical. Toutefois les résultats ne sont pas

\footnotetext{
- Why Some Companies Make the Leap ... and Others Don't, New York, Harper Coollins, 2001.
} 
toujours au bout du chemin et les auteurs signalent bien que «la diversification représente une rupture avec la stratégie habituelle du champion caché, et en change le caractère».

La croissance de ces Champions Cachés repose sur un second pilier : la mondialisation. Celui-ci traduit une approche où les entreprises ont une activité réduite par leur périmètre mais «très ouverte sur la dimension géographique». La mondialisation est devenue une nécessité et une option incontournable d'autant plus que $74 \%$ des dirigeants interrogés indiquent qu'ils ont commencé à exporter dès le de début des activités de leurs entreprises. Les Champions Cachés ont en moyenne vingt-quatre filiales à l'étranger (chiffre élevé en le rapportant à la taille moyenne). Les filiales ne sont pas le seul moyen utilisé. La présence se concrétise par des bureaux, par l'intermédiaire de partenaires, bref «en dépit de leur petite taille et de leurs ressources limitées, beaucoup sont déjà de vraies entreprises mondiales : des Champions Cachés du XXI ${ }^{\circ}$ siècle. Le monde est leur marché et ils y renforcent leur présence avec leurs propres structures ».

De plus, le couple focalisation/mondialisation permet aussi d'avoir l'ambition de développer une marque "globale» car ces entreprises ont une forte image auprès de leurs cibles. A titre d'exemple, Miele, fabricant d'électroménager haut de gamme, a construit une forte image de marque mondiale (42 filiales et $73 \%$ du chiffre d'affaires à l'étranger). Mais il ne faut pas oublier que si la mondialisation est le second pilier de la stratégie, le choix «est un processus long qui s'étend sur plusieurs générations et qui exige une vision et des objectifs à long terme, tout autant qu'une grande persévérance».

Une autre dimension importante pour les Champions Cachés réside dans leur proximité avec les clients. Les auteurs montrent, que nous soyons chez le leader mondial des galettes de silicone hyper-pures ou le leader mondial des logiciels d'assistance aux opérations chirurgicales, que les activités de développements ne peuvent pas se réaliser sans les clients. Ils ont calculé que la part des employés ayant des contacts réguliers avec des clients est cinq fois plus élevé chez un Champion Caché que dans une grande entreprise. La complexité des produits ou des services impose des liens directs. Les clients sont exigeants et ont des besoins complexes (qualité, économies réalisées, ponctualité des livraisons, conseil avant-vente et après vente).

Objectifs ambitieux, focalisation, mondialisation et proximité avec les clients s'avéraient largement insuffisants pour assurer la base du succès si l'on ne tenait pas compte de l'innovation. "Atteindre et maintenir une position de leadership mondial exige une capacité à innover, forte et durable». RUD, leader mondial des chaînes industrielles indique qu' «être le leader de l'innovation technologique a toujours été un élément clé de notre vision et de notre stratégie»; Omicron, leader mondial des microscopes électroniques, précise que «nous ne créons pas de valeur par notre outil de production, mais par l'innovation et le développement. Nos clients honorent notre avance technologique». La force des Champions Cachés est d'être à la fois «orientés technologie» et «orientés marché» $(65 \%$ contre $19 \%$ pour les grands groupes selon les auteurs). Ces Champions Cachés ne recherchent pas les ruptures technologiques ; ils sont nombreux ceux qui réussissent car «ils ne cessent de lancer de petites innovations ».

L'origine des innovations est bien complexe à classer d'autant plus que ces innovations sont diverses et que «la chance ou le hasard ont souvent joué leur rôle». Il semble au moins acquis que «le marché et la solution technologique se sont rencontrés relativement tôt dans le processus». Ainsi, les compétences disponibles en interne et les opportunités de marché seraient à la base d'une grande partie des innovations des Champions Cachés. A cet égard, les exemples fournis par les auteurs sont très intéressants : la machine à rouge-à-lèvres, l'échangeur d'ions, la nourriture pour bébé bio, la nourriture pour poissons d'aquarium pour laquelle le fondateur de Tetra, en rédigeant sa thèse, découvrit qu'il y avait absence de nourriture appropriée : aujourd'hui l'entreprise est leader mondial avec plus de $50 \%$ de part de marché. Le top management a un rôle crucial pour les Champions Cachés. Le fondateur de l'entreprise a souvent un rôle de stimulateur d'innovations, une source d'inspiration, «beaucoup d'idées innovantes naissent de l'observation du client et de sa façon de fonctionner». Pour les auteurs, «le management doit stimuler, de façon active et intense, le processus d'innovation, et doit se poser en promoteur en chef des innovations réalisées. Cette responsabilité exige une bonne proximité avec l'activité et ses détails ».

Les dirigeants et surtout leur personnalité méritent une analyse approfondie. Les auteurs distinguent deux profils : les «entrepreneurs fondateurs» (avec cinq traits : unité entre la personne et l'entreprise, détermination, audace, endurance et persévérance, et capacité à inspirer les autres), et les «entrepreneurs développeurs» (formés dans le monde académique avec diplôme d'université, souvent des études à l'étranger et des expériences dans divers pays et parfois dans d'autres entreprises) qui représentent une autre vision «plus cosmopolite du monde et une urbanité certaine». L'énergie entrepreneuriale a laissé place au professionnalisme managérial. L'étude des auteurs montre que cette génération préserve les forces traditionnelles des Champions Cachés mais que ces forces traditionnelles doivent être combinées avec «la sophistication et l'expérience internationale attendues de l'entrepreneur d'une activité mondiale». De manière générale, ces dirigeants inculquent aux employés une culture d'entreprise basée essentiellement sur la loyauté, la qualification, la motivation, la souplesse et le climat de travail. Les dirigeants des Champions Cachés font tout pour motiver et garder leurs spécialistes d'où un taux d'absentéisme et de turnover des employés le plus bas possible. Les auteurs notent ainsi que le taux moyen de renouvellement des employés des Champions Cachés est extrêmement bas ne dépassant pas 
2,7\% (30,6\% aux Etats-Unis, 9\% en Autriche, $8,8 \%$ en Suisse, 7,3\% en Allemagne et 5,3\% pour une firme comme Daimler.

A partir du moment où «le financement, l'organisation et l'environnement forment la cadre général dans lequel une entreprise, de par ses processus clés, va créer de la valeur pour ses clients », les auteurs ont relevé un financement solide avec un autofinancement dominant concernant les Champions Cachés pour lesquels le financement n'est pas perçu comme une contrainte sur la stratégie. Une simplicité pour l'organisation, avec l'approche « un produit, un marché», s'accompagne de la simplicité des structures, des organisations et des processus. Pour eux, une plus grande complexité organisationnelle menace leurs forces traditionnelles. Ils privilégient donc la décentralisation et la structure en divisions spécifiques à des segments de clients par secteur ou région. Les auteurs relèvent aussi que le degré d'intégration verticale reste très élevé même s'il a tendance à baisser ces dix dernières années notamment pour les activités non critiques de l'entreprise. Ainsi, pour leur cœur de compétences, les Champions Cachés «favorisent un fort degré d'intégration verticale et évitent toute externalisation, afin de maintenir leurs standards de qualités élevés. Ils sont aussi convaincus qu'un avantage concurrentiel réel ne peut s'acheter sur le marché, ne pouvant être créé qu'en interne ». On apprend aussi que ces Champions Cachés sont peu enclins aux alliances stratégiques et ne pensent pas que d'autres entreprises puissent les aider à résoudre leur problème, préférant ainsi «rester maître à bord».

Pour terminer cette analyse documentée, les auteurs nous invitent dans le dernier chapitre - fort instructif - à tirer des enseignements des Champions Cachés. Nous retiendrons notamment :

- Des objectifs ambitieux qui sont le socle des réussites extraordinaires : «le leadership est la capacité à motiver les employés dans le monde entier pour devenir les meilleurs, afin d'être le numéro un mondial de son marché».

- Une culture d'entreprise basée sur la performance et sur des employés motivés et qualifiés : «les employés qui restent apprécient cette culture d'entreprise et offrent en retour loyauté et engagement».

- Une profondeur dans la chaîne de valeur avec une forte intégration verticale : «ce qui rend une entreprise unique vient de ses compétences internes propres et ne peut donc être externalisé».

- Une décentralisation rigoureuse qui peut aller jusqu'à des structures légalement indépendantes donnant ainsi naissance à de nouveaux petits champions cachés : «Décentraliser est le meilleur moyen de préserver les forces et les capacités internes des Champions Cachés, même au sein des structures les plus grandes ou les plus complexes».
- Une focalisation sur un seul domaine d'activité pour ne pas disperser des ressources limitées : «c'est uniquement en concentrant ses ressources sur des objectifs ambitieux que l'on peut atteindre une position d'envergure mondiale».

- Une participation active à la mondialisation qui offre des opportunités de croissance même dans les marchés de niche : «»Afin de saisir ces opportunités, les dirigeants et les employés doivent mettre de côté les différences culturelles. Endurance et persévérance sont nécessaires pour survivre à ce processus de mondialisation qui s'inscrit dans la durée».

- Une stratégie d'innovation qui combine les tendances du marché et les évolutions technologiques permettant ainsi une croissance significative des parts de marchés relatives : "L'innovation est le seul moyen efficace à long terme pour faire face à la concurrence. La capacité à innover est une question de créativité et de qualité des ressources plus qu'une question de moyens financiers ».

- Une proximité du client considérée comme le plus grand atout, loin devant les compétences techniques : «Etre proche de ses clients procure automatiquement un avantage concurrentiel certain. Les meilleurs clients, tout comme les meilleurs concurrents, doivent être systématiquement utilisé comme une source de motivation pour toujours faire mieux ».

Enfin, afin de bien faciliter leur mémorisation, H. Simon et $\mathrm{S}$. Guinchard présentent dans un graphique ces 8 enseignements en trois cercles :

- Dans le premier cercle, le cour, on trouve le leadership aux objectifs ambitieux,

- Le cercle du milieu comprend les compétences internes : la profondeur dans la chaîne de valeur, la décentralisation et la performance des employés,

- Le cercle extérieur prend en compte des variables comme la focalisation, la mondialisation, l'innovation et la proximité des clients.

Il s'agit d'un livre tout à fait enrichissant et nous ne pouvons que saluer la démarche des auteurs qui ont illustré leurs propos et analyses par des données quantitatives et qualitatives rigoureuses, et par nombreux exemples obtenus auprès des dirigeants des Champions Cachés. L'adhésion des auteurs à la stratégie des Champions Cachés est telle que nous avons la nette impression d'un avant et après ces Champions qui servent, si l'on a bien compris, de point de repère à bon nombre d'acteurs. Ainsi, dans cet ouvrage, des enseignements sont proposés également pour les directions de la stratégie (orientation long terme et survie de l'entreprise, éviter les grosses erreurs), les petites entreprises, les entreprises de taille intermédiaire, les grands groupes (les grands champions, les conglomérats diversifiés, la dynamique des entreprises), les candidats à un emploi, les 
dirigeants de pays (pays industrialisés développés et les pays émergents).

Après «Small is beautiful», voici venu le temps des «Hidden Champions are wonderful» ...

A la lecture assidue des 334 pages de ce passionnant ouvrage, des interrogations subsistent toutefois. Comment admettre par exemple que l'absence de l'externalisation et des alliances stratégiques puisse contribuer à la supériorité des Champions Cachés? On ne peut qu'être surpris par ce constat à un moment où la tendance dans la management stratégique est au partage des connaissance, aux réseaux et aux alliances comme l'ont montré plusieurs contributions de la Revue Management International ${ }^{4}$. On aurait souhaité que l'analyse des cœurs de compétences des entreprises analysées soit plus approfondie à partir des outils de la théorie des ressources et des compétences. Enfin, nous regrettons néanmoins que les références aux grands auteurs de la discipline du management stratégique soient souvent absentes. De plus, des comparaisons ou confrontations auraient enrichi les analyses avec une démarche plus comparative entre les différentes catégories d'entreprises (TPE, PME, ETI, GE). Cette démarche aurait eu le mérite de relativiser les stratégies des Champions Cachés pour, peut être, mieux mettre en valeur leur originalité ...

4. Voir notamment les numéros hors série suivants de Management International : "Gestion des connaissances dans la Société et les Organisations», 2012, Vol. 16; «Les alliances asymétriques», 2005, Vol. 10. 